\title{
SUMO-mediated regulation of synaptonemal complex formation during meiosis
}

\author{
Carlos Egydio de Carvalho and Mónica P. Colaiácovo ${ }^{1}$ \\ Department of Genetics, Harvard Medical School, Boston, Massachusetts 02115, USA
}

The propagation of most sexually reproducing species is possible due to a specialized form of cell division known as meiosis, which leads to the formation of haploid gametes that fuse upon fertilization, reconstituting the species ploidy. A hallmark of meiosis is the ability to segregate homologous chromosomes away from each other, thereby reducing the chromosome set by half. Mechanistically, this involves pairing, synapsis, and the reciprocal exchange of genetic material (crossover recombination) between homologous chromosomes during prophase I. These events ensure that homologs remain physically connected even after they desynapse, allowing for their proper alignment at the metaphase plate and subsequent segregation to opposite poles of the spindle during the first meiotic division. Failures in homolog recognition or in maintaining homologous interactions invariably disrupt meiotic segregation and result in aneuploid gametes. The importance of proper homologous segregation is underscored by the infertility, miscarriages, and various birth defects that trace back to errors in single meiotic events in the paternal or maternal germline progenitors (Hassold and Hunt 2001).

Among the various processes that chromosomes undergo during prophase I of meiosis, the establishment of the synaptomenal complex (SC), a proteinaceous framework assembled between homologous chromosomes, is required for the subsequent maintenance of synapsis. While the initial pairing between homologs occurs in the absence of the SC, polymerization of this structure ensures the continuous and stable association (synapsis) along homologous chromosomes throughout pachytene, during which time the completion of reciprocal strand exchange events take place (Page and Hawley 2004).

The link between homologous association and recombination is particularly evident in Saccharomyces cerevisiae, where synapsis ultimately depends on doublestrand break (DSB) formation. Indeed, in yeast chromosomes, the polymerization of the SC initiates at sites undergoing meiotic recombination /Chua and Roeder

${ }^{1}$ Corresponding author.

E-MAIL mcolaiacovo@genetics.med.harvard.edu; FAX (617) 432-7663.

Article is online at http://www.genesdev.org/cgi/doi/10.1101/gad.1457806.
1998) and requires the activities of a DSB-inducing enzyme, as well as of strand invasion/exchange proteins (Giroux et al. 1989; Rockmill et al. 1995; Keeney et al. 1997; Peoples et al. 2002). After DSBs are resolved into either reciprocal crossover or noncrossover repair events, the SC gradually disassembles. The homologs, however, remain associated through chiasmata resulting from the earlier crossover recombination events, underpinned by flanking sister chromatid cohesion.

The functional dependency between the formation/ disassembly of the SC and maturation of recombination intermediates is intuitive if one considers the importance of preventing DNA exchange between nonhomologous chromosomes and assuring the successful segregation of homologous chromosomes away from each other upon the first meiotic division. However, despite a long history of research focused on the SC since its initial description (Fawcett 1956; Moses 1956), the mechanisms of SC assembly and disassembly within the context of other meiotic events still remain incompletely characterized. In this issue of Genes \& Development, new findings by Wang and colleagues (Cheng et al. 2006) reveal a link, in S. cerevisiae, between sumoylation and the regulation of both SC assembly and the propensity of SC proteins to form aggregates known as polycomplexes. They demonstrate that Zip3, a protein involved in the initiation of SC formation, is a SUMO (small ubbiquitinlike modifier) E3 ligase. Moreover, their results suggest that Zip1, a building block of the yeast SC, binds to SUMO-conjugated proteins. These interactions may be important for homology sorting during early prophase, as well as in triggering extensive SC polymerization once homologs are paired during mid-prophase. Apart from introducing sumoylation as a mechanism driving SC polymerization, these findings suggest that SUMO could be similarly involved in the assembly of other complex protein structures.

\section{Structure of the SC in S. cerevisiae}

The SC is composed of a pair of lateral elements connected by transverse filaments that form the central region of this structure (Fig. 1). The lateral elements (LE) 

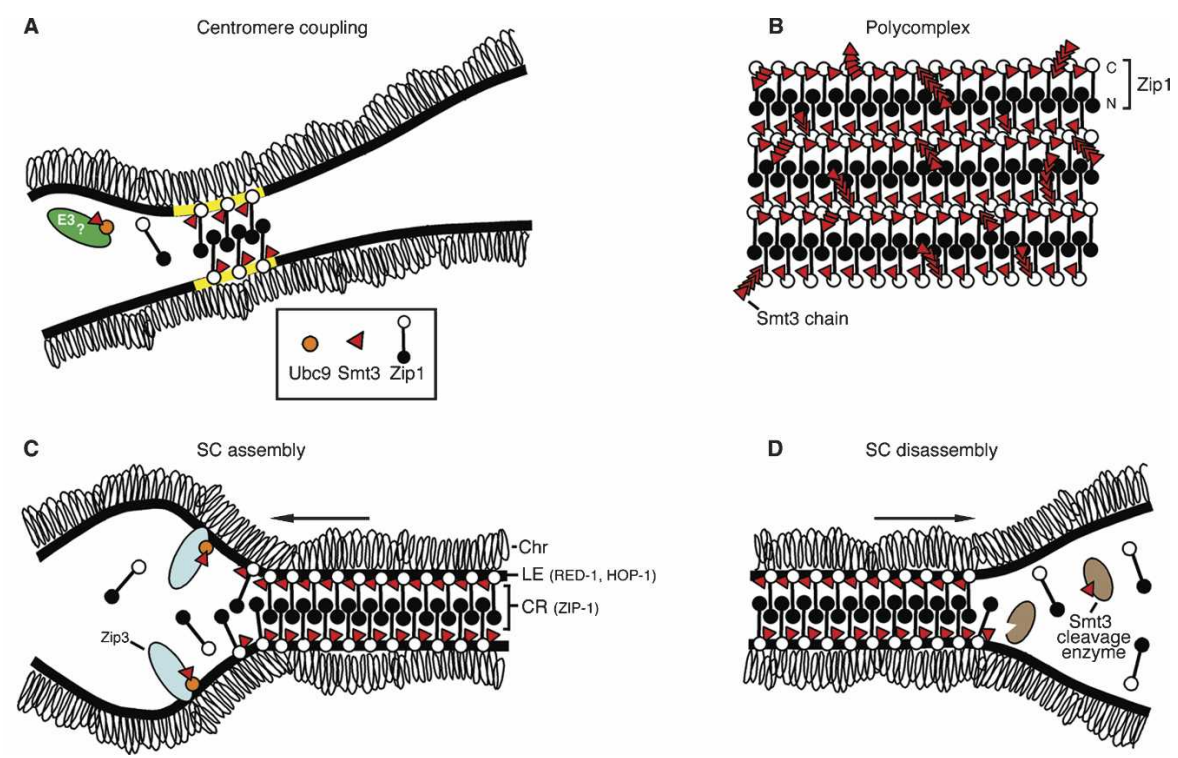

Figure 1. Different modes of Zip1 assembly during yeast meiosis. $(A)$ Homology-independent centromere coupling in yeast may act as a dynamic homology sorting mechanism during early prophase. The presynaptic association between nonhomologous chromosomes via their centromeres or peri-centromeric sequences (yellow) is mediated by Zip1. Zip3-independent E3 ligases, indicated here as "E3?" (green), lead to the formation of Smt3 conjugates that may recruit Zip1 to the centromeres. (B) Zip1 is also observed in aggregates known as polycomplexes, which are particularly evident when SC formation is abrogated. In this context, Smt3 chains (depicted as multiple interconnected red triangles) are associated to the $\mathrm{C}$ terminus of Zip1 in a Zip3-independent fashion. (C) SC assembly occurs through the polymerization of Zip1 homodimers along the interface between homologs where it may associate with Zip3-dependent Smt3 conjugated products. (D) SC disassembly could involve the stepwise dissociation of Smt3 conjugates from axis-associated proteins (e.g., Red1) through the activity of a Smt3 protease or other Smt3-antagozing mechanisms. (Chr) Sister chromatids, (LE) lateral element, (CR) central region.

derive from the original axial elements consisting of proteins, such as Hop1 and Red1, assembled along the axis of each pair of sister chromatids (for review, see Page and Hawley 2004). Meanwhile, proteins such as Zip1, Zip2, and Zip3 are required for the formation of the central region of the SC. In zip1 and zip2 mutants, although normal axial structures form and chromosomes initially pair, they fail to synapse (Sym et al. 1993; Chua and Roeder 1998), while in zyp3 mutants, formation of the $\mathrm{SC}$ is both delayed and incomplete (Agarwal and Roeder 2000).

Zip1 is a structural component of the SC consisting of an extended coiled-coil domain flanked by globular domains. It is a transverse filament protein that forms homodimers that interact head to head via their N-terminal globular domains, while associating with the LEs via their C-terminal globular domains, thus spanning the width between homologous axes (Sym et al. 1993; Dobson et al. 1994; Liu et al. 1996; Schmekel et al. 1996; Dong and Roeder 2000).

Zip2 and Zip3 are proteins required for Zip1 polymerization on chromosomes (Agarwal and Roeder 2000). SC formation in yeast initiates specifically on the subset of DSB sites undergoing crossover recombination. This involves the "synapsis initiation complex," which includes Zip1, Zip2, Zip3, and the activity of the yeast meiotic recombination machinery at sites of axial associations (Peoples et al. 2002; Fung et al. 2004). Zip3 acts as a link between initiation of synapsis and meiotic re- combination by recruiting Zip2, stabilizing its association to sites of synapsis initiation, and interacting with a series of early (Mre11, Rad51, Rad57) and late (Msh4, Msh5) recombination proteins (Agarwal and Roeder 2000). Furthermore, Zip3 physically interacts with Zip2 and Zip1 and acts upstream from both (Agarwal and Roeder 2000). Altogether, the localization of Zip3 and its protein interactions suggest that it may mediate the cross-talk between SC proteins and meiotic recombination during synapsis initiation in yeast. The studies by Cheng et al. (2006) now place Zip3 as a SUMO E3 ligase and Zip1 as either binding SUMO-conjugated products during SC polymerization or as a target for noncovalently bound SUMO modification when SC formation is abrogated, further suggesting a fine-tuned regulation of SC formation.

\section{Polycomplex formation}

Transverse filament proteins have a propensity for selfassembly, leading to the formation of aggregates known as polycomplexes (Fig. 1B). These aggregates are observed in wild type mostly upon SC disassembly (Zickler and Kleckner 1998). However, their formation is exacerbated in various organisms under situations where DNA replication, synapsis, or recombination are perturbed, as well as when transverse filament proteins are overexpressed in meiosis or expressed mitotically (Sym and Roeder 1995; Yuan et al. 1996; Zickler and Kleckner 
1998; Ollinger et al. 2005). Interestingly, analysis via electron microscopy revealed that proteins within the polycomplex acquire an organization that resembles that observed in the SC (Dong and Roeder 2000; Ollinger et al. 2005). Presumably, polycomplexes reflect the intrinsic ability of central region proteins to self-assemble in conditions where SC polymerization is precluded (Zickler and Kleckner 1999). How the delicate balance between self-assembly and SC assembly is regulated at the biochemical level remained elusive until now.

\section{Sumoylation and its functions}

The conjugation of SUMO to target proteins, or sumoylation, is a highly conserved and reversible post-translational modification. It modulates protein-protein and protein-DNA interactions and is involved in regulating a variety of cellular processes such as nuclear transport, signal transduction, stress response, and cell cycle progression (Muller et al. 2001; Schwarz and Hochstrasser 2003; Hay 2005). Interestingly, while ubiquitination usually targets substrates for degradation via the $26 \mathrm{~S}$ proteosome, the addition of SUMO conjugates appears to promote stability and regulate the subcellular localization of its targets (Muller et al. 2001).

Sumoylation unfolds via a three-enzymatic-step pathway analogous to (but distinct from) ubiquitination (Fig. 2; for review, see Muller et al. 2001; Melchior et al. 2003; Gill 2004; Hay 2005). Upon proteolytic maturation, the yeast SUMO protein, Smt3, is activated through the formation of a thioester bond between its $\mathrm{C}$ terminus and an E1 SUMO-specific activating complex (Uba2/Aos1) before it is transferred to the E2-conjugating enzyme, Ubc9. Ubc9 ultimately catalyzes the covalent link between a C-terminal glycine in Smt3 and a lysine residue present in the sumoylation consensus sequences of the target protein. In vitro studies showed that Ubc9 supports substrate recognition and Smt-3 ligation (Bencsath et al. 2002). However, E3 ligases play an important role in enhancing substrate identification and specificity. Moreover, it is possible that E3 ligases promote SUMO conjugation of lysines located in nonconsensus sequences of the target proteins (Melchior et al. 2003).

SUMO-specific E3 ligases were the last enzymes in the sumoylation pathway to be discovered. To date, three distinct classes of SUMO E3 ligases have been identified. Among these, E3 ligases belonging to the PIAS (Protein Inhibitor of Activated STAT) protein family share a RING domain essential for E3 ligase activity (for review, see Melchior et al. 2003). The yeast Siz1 and Siz2 proteins, required for cytokinesis, are SUMO E3 ligases that bind Ubc9 and septins (cytoskeletal GTP-binding proteins that constitute the main sumoylation targets in $S$. cerevisiae). Both Siz1 and Siz2 contain the signature RING domain and are redundantly involved in SUMO conjugation of most yeast sumoylation targets at different stages of the cell cycle (Johnson and Gupta 2001; Takahashi et al. 2001). A link between chromosome structure and SUMO was recently established with the identification of E3 ligase activity for the structural

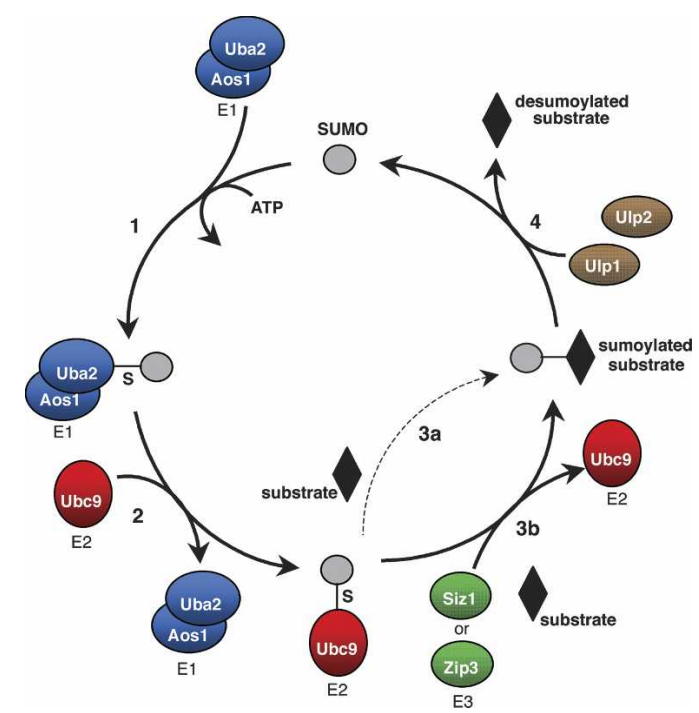

Figure 2. The SUMO conjugation and deconjugation pathway in yeast. SUMO activation is achieved through ATP-dependent formation of a thioester bond by the $\mathrm{E} 1$ heterodimer complex Uba2/Aos1 (blue) (step 1). This is followed by conjugation to the E2 enzyme Ubc9 (red) (step 2). Although Ubc9-SUMO is capable of targeting and catalyzing the covalent addition of SUMO molecules to certain substrates (step 3a), E3 enzymes such as Siz1 (green) enhance both substrate identification and specificity (step 3b). While E3 ligases such as Siz1 and Siz2 act during early prophase of meiosis I, ZIP3 (green) acts during SC formation in mid-prophase. Desumoylation relies on the activity of the yeast ULP proteases, Ulp1 and Ulp2 (brown), which remove the covalently linked SUMO from the C terminus of targeted substrates (step 4).

maintenance of chromosomes (SMC)-associated protein Mms21 in yeast and humans (Potts and Hongtao 2005; Zhao and Blobel 2005). Searching for other RING domain-containing proteins in yeast, Cheng et al. (2006) identified the SC component Zip3 as a putative SUMO E3-ligase.

\section{SUMO and meiosis}

Several observations suggest a possible role for SUMO in chromatin remodeling and DNA repair during meiosis. During early sporulation in yeast, mRNA levels of the desumoylation enzyme Ulp2 increase, presumably reflecting a specific need for deconjugation. ulp2 mutants show severe sporulation defects that are mirrored by changes in Smt3 conjugation during meiosis ( $\mathrm{Li}$ and Hochstrasser 2000; Muller et al. 2001). In humans, the DSB repair proteins RAD-51 and RAD-52 interact with both SUMO and the E2-conjugating enzyme UBC-9 in yeast two-hybrid assays (Shen et al. 1996). Moreover, the human DNA topoisomerases I and II are sumoylated in response to DNA damage (Mao et al. 2000a,b). However, despite the previously observed colocalization of mammalian UBC-9 and RAD-51 proteins at the SC (Kovalenko et al. 1996), the connection between SUMO and SC assembly had not been fully established until now. 
Cheng et al. (2006) observed that Smt3 conjugates formed foci along chromosomes during early prophase in a Zip3-independent manner. However, they localized continuously along chromosomes after mid-prophase, colocalizing with Zip1 during pachytene in a Zip3-dependent manner. Moreover, when SC formation was impaired, either in a zip3 mutant or due to the lack of initiation of meiotic recombination, Smt3 conjugates colocalized with Zip1 on polycomplexes. These observations not only demonstrate that Smt3 conjugates are components of the SC and of polycomplexes but also suggest a connection between SUMO and Zip3 in the regulation of SC formation. Interestingly, in an ulp2 zip3 double mutant, the absence of the Smt3 deconjugating enzyme leads to persistence of the early Smt3/Zip1 foci on chromosomes and, as in a zip3 mutant, lack of SC formation. However, Zipl-mediated SC formation is still observed in $>25 \%$ of ulp 2 cells. Altogether, these observations suggest two distinct temporal modes of action for Smt3-conjugated products during meiosis. First, during early prophase, Smt3-conjugated products are involved in the formation of foci containing Zip1 that have been recently proposed to act in centromere coupling (Tsubouchi and Roeder 2005). This presynaptic association between meiotic chromosomes in yeast is independent of the recombination machinery and of chromosome homology. It has been proposed that during this process, chromosomes switch partners via dynamic centromeric connections until homologous associations are established and DSB-dependent pairing initiates (Tsubouchi and Roeder 2005). A second mode of action for Smt3-conjugated products would come into play during mid-prophase, in SC formation via Zip3-dependent SUMO modifications.

Through combined cytological and biochemical approaches, Wang and colleagues (Cheng et al. 2006) observed the accumulation of Smt3-conjugated products in zip3 mutants but not in other mutants defective in synapsis, meiotic recombination, or meiotic cell cycle regulation. Moreover, the investigators determined that Zip3 exhibits Smt3 ligase activity in vitro and interacts specifically with Smt3 and Ubc9 as shown by yeast twohybrid. They also observed a probable noncovalent interaction between Zip1 and Smt3 during meiosis. In wildtype cells, Zip1 binds Smt3 through a Smt3 binding motif (SBM) located in its C-terminal domain. Surprisingly, both yeast two-hybrid and in vitro binding assays suggest that the C-terminal domain of Zip1 has a stronger affinity to Smt3 polymeric chains compared to Smt3 monomers. This is particularly revealing since polycomplexes formed in the absence of Zip3 consist of Smt3 polymeric chains associated to Zip1.

Altogether, these results suggest that SUMO E3 ligases are essential for the early organization of the SC, perhaps by stabilizing Zip1 affinity for Smt3-conjugated products. It also supports the interpretation that the SC arises by controlling the self-assembly tendencies of its proteins, thereby preventing the formation of polycomplexes. Expectedly, both the activity and localization of Zip3 during meiosis are under stringent control. Zip3 itself is conjugated to Smt3 in early prophase (by another, as of yet not identified, E3-ligase) and is phosphorylated as it loads onto chromosomes. Phosphorylation, as a post-translational mechanism to control transient localization and hence activity of SUMO E3 activity, has been previously suggested for Sizl. Upon phosphorylation, Sizl translocates from the nucleus to the bud-neck region during mitosis (Johnson and Gupta 2001). Interestingly, phosphorylation of SUMO targets appears to regulate substrate conjugation (Muller et al. 2000; Yang et al. 2003; Kang et al. 2006). As Zip3 is also a substrate for sumoylation, it is possible that its transient association with Smt3 is regulated through phosphorylation.

Consistent with its previously characterized associations with the yeast repair proteins, the role of Zip3 in SC assembly also depends on DSBs, placing it downstream from the initiation of recombination. Moreover, Zip3 is able to recruit Smt3 to chromosomes in the absence of either Zip1 or Zip2, although it is not necessary for the initial Zip1/Smt3 foci observed in early prophase. These results, altogether, lead the authors to propose the following model for the fine-tuned, sumoylation-dependent, regulation of SC assembly and polycomplex formation. First, through the activity of Zip3-independent E3 ligases expressed during early prophase, Zipl is targeted to chromosomes and participates in nonhomologous centromere coupling (Fig. 1A). Later, at the time of SC nucleation and polymerization, Zip1 associates with Zip3-dependent Smt3 conjugated products assembled onto chromosomes (Fig. 1C). These two stages are separated by a brief desumoylation interval that requires Ulp2 activity and results in the disappearance of the Zip1/Smt3 foci as prophase advances and homologs pair. However, in instances where progression to SC assembly is perturbed (such as in a zip3 mutant), polycomplexes form, consisting of polymeric Smt3 chains associated to Zip1 (Fig. 1B). The complexity of Zip1-Smt3 transient associations, the requirement of Zip1 for crossover maturation, the involvement of Zip3, and the presence of other sumoylated LE-associated proteins such as Top2, Red1, and Pds5 (Stead et al. 2003; Cheng et al. 2006; Takahashi et al. 2006) strongly suggest that the functional consequences of SUMO conjugates on yeast meiotic chromosomes are extensive. The dissection of these regulatory webs will likely expose further molecular interactions between SC assembly/disassembly and the components of various other events unfolding during meiosis (e.g., DNA repair machinery, condensins, and cohesins). An analogous situation is observed with SUMO's more famous "relative," ubiquitin.

\section{Controlling SC dynamics}

The ubiquitination machinery is essential for monitoring DNA damage and engaging in DNA repair. During replication, template switch in response to DNA damage is triggered by ubiquitination of PCNA /proliferating cell nuclear antigen) by E2-E3 complexes belonging to the Rad6 epistasis group in yeast (Motegi et al. 2006). Curi- 
ously, in mutants deficient in this surveying pathway, PCNA is sumoylated by Sizl at the same lysine residue targeted for ubiquitination. Instead of aborting replication of the damaged template, SUMO-conjugated PCNA recruits the helicase Srs2 to stalled replication forks, ultimately initiating repair through homologous recombination. The consequence is the accumulation of chromosomal abnormalities and genomic instability (Motegi et al. 2006). In this context, ubiquitin and SUMO conjugation behave as opposing signals in the suppression or generation of accumulated DNA damage, respectively. Similar examples of substrate competition between ubiquitin and SUMO in regulating different physiological outcomes indicate that these analogous post-translational systems might have evolved in many situations to behave antagonistically (Desterro et al. 1998; Buschmann et al. 2000). This is particularly intriguing in light of the SC defects observed in knockout mice for the mammalian Rad6 homolog HR6B. Lack of this E2 ubiquitin conjugation enzyme in mice causes male infertility due to a defect in meiotic prophase during spermatogenesis (Baarends et al. 2003). In addition to increased primary spermatocyte apoptosis, the SCs of pachytene nuclei are longer and thinner, presumably due to a loose chromatin-axis association. More importantly, the SC is absent in the regions next to the telomeres. Interestingly, this pattern is normally seen in wild-type nuclei late in diplotene, a sign that the SC of HR6 $\mathrm{B}^{-/-}$animals might be disassembling prematurely (Baarends et al. 2003). On the other hand, expression analysis in mouse and human testis showed that SUMO is present in sex bodies during chromatin condensation, when it could facilitate synapsis between the $\mathrm{X}$ and $\mathrm{Y}$ chromosomes (Vigodner and Morris 2005; Vigodner et al. 2005).

SUMO and ubiquitin are also deeply involved in the dynamics of chromosome segregation in mitosis. In this case, however, there is evidence that both systems promote dissociation of cohesion between sisters. The APCmediated polyubiquitination of Securin triggers metaphase/anaphase transition by activating Separase, whereas desumoylation of centromeric components is necessary for maintenance of sister chromatid cohesion (Bachant et al. 2002; Yu 2002; Stead et al. 2003). In addition, SUMO conjugation has been detected for all cohesin/condensin SMC complexes in yeast (Lee and O'Connel 2006). Mms21, a non-SMC component of the cohesin complex, was recently shown to be a SUMO ligase that recognizes its own complex as a substrate (Lee and $\mathrm{O}^{\prime}$ Connel 2006). Mms21-mediated sumoylation most likely controls chromosome localization of the complex, possibly also affecting its role in recombination.

It will be important to examine how widespread are the roles of sumoylation and ubiquitination of SC proteins across phyla. An example is the Caenorhabditis elegans Zip3 homolog, ZHP-3, which is involved in crossover recombination and, consequently, chiasma formation in a SC-dependent manner (Jantsch et al. 2004). While this reveals a conserved role for Zip3 homologs in regulating meiosis, unlike the yeast zip3 mu- tant, SC formation is not impaired in a $z h p-3$ mutant. Given that SC formation is DSB independent in C. elegans (Dernburg et al. 1998), and given the apparent involvement of different E3 ligases between early and midprophase events in yeast (Cheng et al. 2006), it is possible that the link between sumoylation and SC formation is exerted by an earlier acting E3 ligase in worms. The intricacies of such regulation remain to be determined. It is, however, tempting to conceive a link between the mechanisms of sumoylation and ubiquitination in the assembly (SUMO) and disassembly (ubiquitin) dynamics of the SC. Alternatively, desumoylation may play a key role in dismantling the SC, either by reducing the affinity of Zip1 to lateral element components or by actively triggering its removal from the central region of the SC (Fig. 1D). Identifying additional meiotic substrates undergoing such post-translational modifications and characterizing their kinetics during SC assembly and disassembly will be essential to unravel how these dynamics are regulated.

\section{SUMO and the assembly of complex cellular structures}

The finding by Wang and colleagues that SUMO participates in the regulation of SC assembly is not only of tremendous impact to the field of meiosis but also raises the possibility that sumoylation controls the organization of other transient cellular structures. Although SUMO has been implicated in a variety of regulatory mechanisms such as nuclear transport and transcriptional regulation (Muller et al. 2001), a global role in assembly and turnover of macromolecular structures such as the SC is far less known. Nevertheless, SUMO conjugation seems to be required for assembly and structural integrity of nuclear bodies and viral nucleocapsids (Ishov et al. 1999; Maeda et al. 2003). In particular, the orderly assembly of proteins into the complex multimeric structures of some Hantavirus nucleocapsids appears to involve sumoylation of the individual monomers by the host machinery. As with the yeast SC, this is accomplished by targeting SUMO-modified substrates to the site of assembly (Maeda et al. 2003). Whether SUMO-regulated formation of macromolecular structures is widespread to other cellular structures remains to be investigated.

\section{Acknowledgments}

We thank Grace Gill and Keith Blackwell for critical reading of this manuscript. This work was supported by grants from the National Institutes of Health (GM 072551) and the March of Dimes Birth Defects Foundation (5-FY05-1214) to M.P.C.

\section{Note added in proof}

Since the submission of this perspective article, the analysis of SC formation in an $u b c 9$ mutant in budding yeast has also suggested a relationship between sumoylation and synapsis (Hooker and Roeder 2006). 


\section{References}

Agarwal, S. and Roeder, G.S. 2000. Zip3 provides a link between recombination enzymes and synaptonemal complex proteins. Cell 102: 245-255.

Baarends, W.M., Wassenaar, E., Hoogerbrugge, J.W., van Cappellen, G., Roest, H.P., Vreeburg, J., Ooms, M., Hoeijmakers, J.H.J., and Grootegoed, A. 2003. Loss of HR6B ubiquitinconjugating activity results in damaged synaptonemal complex structure and increased crossing-over frequency during male meiotic prophase. Mol. Cell. Biol. 23: 1151-1162.

Bachant, J., Alcasabas, A., Blat, Y., Kleckner, N., and Elledge, S.J. 2002. The SUMO-1 isopeptidase Smt4 is linked to centromeric cohesion through SUMO-1 modification of DNA topoisomerase II. Mol. Cell 9: 1169-1182.

Bencsath, K.P., Podgorski, M.S., Pagala, V.R., Slaughter, C.A., and Schulman, B.A. 2002. Identification of a multifunctional binding site on Ubc9p required for Smt3p conjugation. $J$. Biol. Chem. 227: 47938-47945.

Buschmann, T., Fuchs, S.Y., Lee, C.G., Pan, Z.Q., and Ronai, Z. 2000. SUMO-1 modification of Mdm2 prevents its self-ubiquitination and increases Mdm2 ability to ubiquitinate p53. Cell 101: 753-762.

Cheng, C.-H., Lo, Y.-H., Liang, S.-S., Ti, S.-C., Lin, F.-M., Yeh, C.-H., Huang, H.-Y., and Wang, T.-F. 2006. SUMO modifications control assembly of synaptonemal complex and polycomplex in meiosis of Saccharomyces cerevisiae. Genes \& Dev. (this issue).

Chua, P.R. and Roeder, G.S. 1998. Zip2, a meiosis-specific protein required for the initiation of chromosome synapsis. Cell 93: 349-359.

Dernburg, A.F., McDonald, K., Moulder, G., Barstead, R., Dresser, M., and Villeneuve, A.M. 1998. Meiotic recombination in C. elegans initiates by a conserved mechanism and is dispensable for homologous chromosome synapsis. Cell 94: 387-398.

Desterro, J.M., Rodriguez, M.S., and Hay, R.T. 1998. SUMO-1 modification of $1 \kappa \mathrm{B} \alpha$ inhibits NF-кB activation. Mol. Cell 2: 233-239.

Dobson, M.J., Pearlman, R.E., Karaiskakis, A., Spyropoulos, B., and Moens, P.B. 1994. Synaptonemal complex proteins: Occurrence, epitope mapping and chromosome disjunction. J. Cell Sci. 107: 2749-2760.

Dong, H. and Roeder, G.S. 2000. Organization of the yeast Zip1 protein within the central region of the synaptonemal complex. J. Cell Biol. 148: 417-426.

Fawcett, D.W. 1956. The fine structure of chromosomes in the meiotic prophase of vertebrate spermatocytes. I. Biophys. Biochem. Cytol. 2: 403-406.

Fung, J.C., Rockmill, B., Odell, M., and Roeder, G.S. 2004. Imposition of crossover interference through the nonrandom distribution of synapsis initiation complexes. Cell 116: $795-$ 802.

Gill, G. 2004. SUMO and ubiquitin in the nucleus: Different functions, similar mechanisms? Genes \& Dev. 18: 20462059.

Giroux, C.N., Dresser, M.E., and Tiano, H.F. 1989. Genetic control of chromosome synapsis in yeast meiosis. Genome 31: 88-94.

Hassold, T. and Hunt, P. 2001. To err (meiotically) is human: The genesis of human aneuploidy. Nat. Rev. Genet. 2: 280291.

Hay, T. 2005. SUMO: A history of modification. Mol. Cell 18: $1-12$.

Hooker, G.W. and Roeder, G.S. 2006. A role for SUMO in meiotic chromosome synapsis. Curr. Biol. 16: 1238-1243.
Ishov, A.M., Sotnikov, A.G., Negorev, D., Vladimirova, O.V., Neff, N., Kamitani, T., Yeh, E.T.H., Strauss III, J.F., and Maul, G.G. 1999. PML is critical for ND10 formation and recruits the PML-interacting protein Daxx to this nuclear structure when modified by SUMO-1. J. Cell Biol. 147: 221223.

Jantsch, V., Pasierbek, P., Mueller, M.M., Schweizer, D., Jantsch, M., and Loidl, J. 2004. Targeted gene knockout reveals a role in meiotic recombination for ZHP-3, a Zip3related protein in Caenorhabditis elegans. Mol. Cell. Biol. 24: 7998-8006.

Johnson, E.S. and Gupta, A.A. 2001. An E3-like factor that promotes SUMO conjugation to the yeast septins. Cell 106: 735-744.

Kang, K., Gocke, C.B., and Yu, H. 2006. Phosphorylation-facilitated sumoylation of MEF2C negatively regulates its transcriptional activity. BMC Biochem. 7: 5.

Keeney, S., Giroux, C.N., and Kleckner, N. 1997. Meiosis-specific DNA double-strand breaks are catalyzed by Spo11, a member of a widely conserved protein family. Cell 88: $375-$ 384 .

Kovalenko, O.V., Plug, A.W., Haaf, T., Gonda, D.K., Ashley, T., Ward, D.C., Radding, C.M., and Golub, E.I. 1996. Mammalian ubiquitin-conjugating enzyme Ubc9 interacts with Rad51 recombination protein and localizes in synaptonemal complexes. Proc. Nat1. Acad. Sci. 93: 2958-2963.

Lee, K.M. and O'Connel, M.J. 2006. A new SUMO ligase in the DNA damage response. DNA Repair (Amst.) 5: 138-141.

Li, S.J. and Hochstrasser, M.A. 2000. The yeast ULP2 (SMT4) gene encodes a novel protease specific for the ubiquitin-like Smt3 protein. Mol. Cell. Biol. 20: 2367-2377.

Liu, J.G., Yuan, L., Brundell, E., Bjorkroth, B., Daneholt, B., and Hoog, C. 1996. Localization of the N-terminus of SCP1 to the central element of the synaptonemal complex and evidence for direct interactions between the N-termini of SCP1 molecules organized head-to-head. Exp. Cell Res. 226: 1119.

Maeda, A., Lee, B.H., Yoshimatsu, K., Saijo, M., Kurane, I., Arikawa, J., and Morikawa, S. 2003. The intracellular association of the nucleocapsid protein (NP) of Hantaan virus (HTNV) with small ubiquitin-like modifier-1 (SUMO-1) conjugating enzyme 9 (Ubc9). Virology 305: 288-297.

Mao, Y., Sun, M., Desai, S.D., and Liu, L.F. 2000a. SUMO-1 conjugation to topoisomerase I: A possible repair response to topoisomerase-mediated DNA damage. Proc. Natl. Acad. Sci. 97: 4046-4051.

Mao, Y., Desai, S.D., and Liu, L.F. 2000b. SUMO-1 conjugation to human DNA topoisomerase II isozymes. J. Biol. Chem. 275: 26066-26073.

Melchior, F., Schergaut, M., and Pichler, A. 2003. SUMO: Ligases, isopeptidases and nuclear pores. Trends Biochem. Sci. 28: 612-618.

Moses, M.J. 1956. Chromosomal structures in crayfish spermatocytes. J. Biophys. Biochem. Cytol. 2: 215-218.

Motegi, A., Kuntz, K., Majeed, A., Smith, S., and Myung, K. 2006. Regulation of gross chromosomal rearrangements by ubiquitin and SUMO ligases in Saccharomyces cerevisiae. Mol. Cell. Biol. 26: 1424-1433.

Muller, S., Berger, M., Lehembre, F., Seeler, J.S., Haupt, Y., and Dejean, A. 2000. C-Jun and p53 activity is modulated by SUMO-1 modification. J. Biol. Chem. 275: 13321-13329.

Muller, S., Hoege, C., Pyrowolakis, G., and Jentsch, S. 2001. Sumo, ubiquitin's mysterious cousin. Nat. Rev. Mol. Cell Biol. 2: 202-210.

Ollinger, R., Alsheimer, M., and Benavente, R. 2005. Mammalian protein SCP1 forms synaptonemal complex-like struc- 
ture in the absence of meiotic chromosome. Mol. Biol. Cell 16: 212-217.

Page, P.L. and Hawley, S. 2004. The genetics and molecular biology of the synaptonemal complex. Annu. Rev. Cell Dev. Biol. 20: 525-558.

Peoples, T.L., Dean, E., Gonzalez, O., Lambourne, L., and Burguess, S.M. 2002. Close, stable homolog juxtaposition during meiosis in budding yeast is dependent on meiotic recombination, occurs independently of synapsis, and is distinct from DSB-independent pairing contacts. Genes \& Dev. 16: 1682-1695.

Potts, P.R. and Hongtao, Y. 2005. Human MMS21/NSE2 is a SUMO ligase required for DNA repair. Mol. Cell. Biol. 25: 7021-7032.

Rockmill, B., Sym, M., Scherthan, H., and Roeder, G.S. 1995. Roles for two RecA homologs in promoting chromosome synapsis. Genes \& Dev. 9: 2684-2685.

Schmekel, K., Meuwissen, R.L., Dietrich, A.J., Vink, A.C., van Marle, J., van Veen, H., and Heyting, C. 1996. Organization of SCP1 protein molecules within synaptonemal complexes of the rat. Exp. Cell Res. 226: 20-30.

Schwarz, D.C. and Hochstrasser, M. 2003. A superfamily of protein tags: Ubiquitin, SUMO and related modifiers. Trends Biochem. Sci. 28: 321-328.

Shen, Z., Pardington-Purtymun, P.E., Comeaux, J.C., Moyzis, R.K., and Chen, D.J. 1996. UBL1, a human ubiquitin-like protein associating with human RAD51.RAD52 proteins. Genomics 36: 271-279.

Stead, K., Aguilar, C., Hartman, T., Drexel, M., Meluh, P., and Guacci, V. 2003. Pds5p regulates the maintenance of sister chromatid cohesion and is sumoylated to promote the dissolution of cohesion. J. Cell Biol. 163: 729-741.

Sym, M. and Roeder, G.S. 1995. Zip-1-induced changes in synaptonemal complex structure and polycomplex assembly. $J$. Cell Biol. 128: 455-466.

Sym, M., Engebrecht, J., and Roeder, G.S. 1993. Zip1 is a synaptonemal complex protein required for meiotic chromosome synapsis. Cell 72: 365-378.

Takahashi, Y., Kahyo, T., Toh-E, A., Yasuda, H., and Kikuchi, Y. 2001. Yeast Ull1/Siz1 is a novel SUMO1/Smt3 ligase for septin components and functions as an adaptor between conjugating enzymes and substrates. J. Biol. Chem. 276: 4897348977.

Takahashi, Y., Yong-Gonzalez, V., Kikuchi, Y., and Strunnikov, A. 2006. SIZ1/SIZ2 control of chromosome transmission fidelity is mediated by the sumoylation of topoisomerase II. Genetics 172: 783-794.

Tsubouchi, T. and Roeder, G.S. 2005. A synaptonemal complex protein promotes homology-independent centromere coupling. Science 308: 870-873.

Vigodner, M. and Morris, P.L. 2005. Testicular expression of small ubiquitin-related modifier-1 (SUMO-1) supports multiple roles in spermatogenesis: Silencing of sex chromosomes in spermatocytes, spermatid microtubule nucleation, and nuclear reshaping. Dev. Biol. 282: 480-492.

Vigodner, M., Ishikawa, T., Schlegel, P.N., and Morris, P.L. 2005. SUMO-1, human male germ cell development, and the androgen receptor in the testis of men with normal and abnormal spermatogenesis. Am. J. Physiol. Endocrinol. Metab. 290: E1022-E1033.

Yang, S.H., Jaffray, E., Hay, R.T., and Sharrocks, A.D. 2003. Dynamic interplay of the SUMO and ERK pathways in regulating Elk-1 transcriptional activity. Mol. Cell 12: 63-74.

$\mathrm{Yu}, \mathrm{H} .2002$. Regulation of APC-Cdc20 by the spindle checkpoint. Curr. Opin. Cell Biol. 14: 706-714.

Yuan, L., Brundell, E., and Hoog, C. 1996. Expression of the meiosis-specific synaptonemal complex protein 1 in a heterologous system results in the formation of large protein structures. Exp. Cell Res. 229: 272-275.

Zhao, X. and Blobel, G. 2005. A SUMO ligase is part of a nuclear multiprotein complex that affects DNA repair and chromosomal organization. Proc. Natl. Acad. Sci. 102: 4777-4782.

Zickler, D. and Kleckner, N. 1998. The leptotene-zygotene transmission of meiosis. Annu. Rev. Genet. 32: 619-697.

- 1999. Meiotic chromosomes: Integrating structure and function. Annu. Rev. Genet. 33: 603-754. 


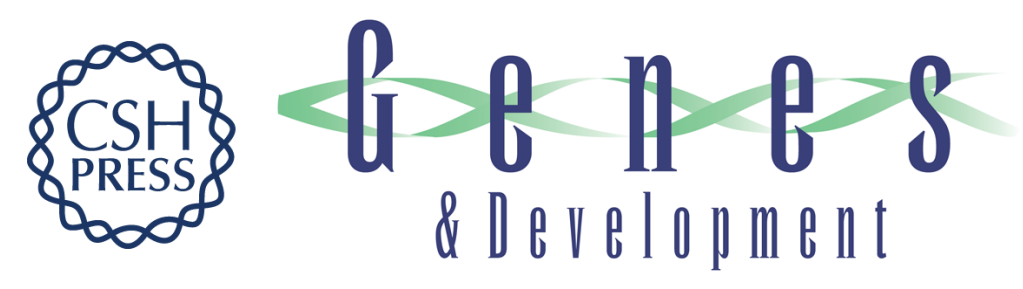

\section{SUMO-mediated regulation of synaptonemal complex formation during meiosis}

Carlos Egydio de Carvalho and Mónica P. Colaiácovo

Genes Dev. 2006, 20:

Access the most recent version at doi:10.1101/gad.1457806

\section{Related Content SUMO modifications control assembly of synaptonemal complex and polycomplex in meiosis of Saccharomyces cerevisiae \\ Chung-Hsu Cheng, Yu-Hui Lo, Shu-Shan Liang, et al. \\ Genes Dev. UNKNOWN , 2006 20: 2067-2081 \\ References This article cites 56 articles, 24 of which can be accessed free at: \\ http://genesdev.cshlp.org/content/20/15/1986.full.html\#ref-list-1 \\ Articles cited in: \\ http://genesdev.cshlp.org/content/20/15/1986.full.html\#related-urls \\ License \\ Email Alerting \\ Receive free email alerts when new articles cite this article - sign up in the box at the top Service

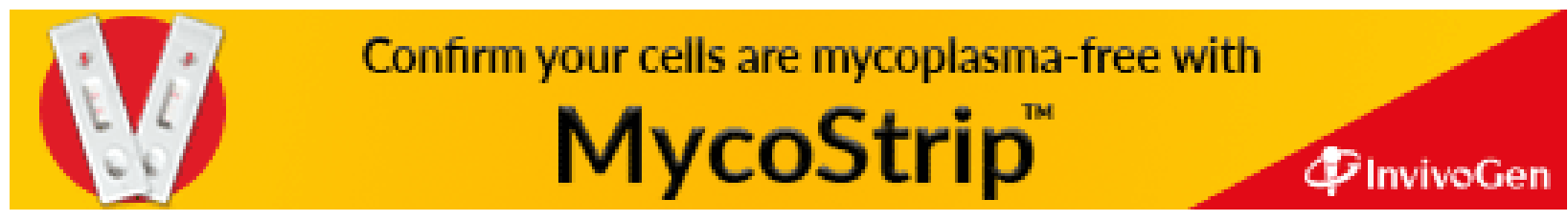

